\title{
Composición y riqueza de odonatos en tres humedales artificiales suburbanos en Cajicá, Cundinamarca, Colombia
}

\author{
Odonata composition and richness in three artificial, suburban \\ wetlands in Cajicá, Cundinamarca, Colombia
}

\author{
José Alejandro Cuéllar-Cardozo ${ }^{1}$, Erika Arteaga-Guzmán ${ }^{1}$, Laura Bernal-Arriero, \\ Aura Campo-Garnica ${ }^{1}$, Diana Capera ${ }^{1}$, Caroline Ocampo ${ }^{1}$, Mónica Ortiz-Ruiz' ${ }^{1}$, \\ Sebastián Rincón-Aceldas ${ }^{1}$, Francisco Sánchez ${ }^{1,2}$
}

\section{Resumen}

El paisaje de la Sabana de Bogotá ha sido altamente modificado y se han creado humedales artificiales con diferentes propósitos, pero poco se sabe sobre su biodiversidad. Objetivo: Comparar la composición y riqueza específica de odonatos en tres humedales artificiales en el campus de la Universidad Militar Nueva Granada en Cajicá, Cundinamarca, Colombia. Metodología: Se capturaron adultos con redes entomológicas durante marzo y abril de 2015, en un humedal cerca de la ronda del río Bogotá, uno temporal rodeado por pastizales y uno rodeado por edificios y pastos con el fondo cubierto por geomembrana. Resultados: Se recolectaron 817 individuos pertenecientes a las siguientes especies: Rhionaeschna marchali (Aeshnidae), Enallagma civile (Coenagrionidae), Ischnura cruzi (Coenagrionidae), Ischnura sp., Mesamphiagrion laterale (Coenagrionidae) y Erythrodiplax fusca (Libellulidae). El humedal de ronda de río y el humedal temporal compartieron cuatro especies y presentaron mayor similitud entre ellos que con el humedal con geomembrana. Hubo una asociación entre la frecuencia de especies de Odonata y cada uno de los humedales; una especie fue exclusiva del humedal de ronda de río ( $R$. marchali) y otra fue exclusiva del humedal temporal (Ischnura sp.). No hubo diferencias entre la riqueza total estimada entre el humedal de ronda y el temporal, y ambos tuvieron una riqueza mayor que el humedal con geomembrana. Conclusión: Los resultados sugieren que las diferencias entre los humedales afectan la composición, frecuencia y riqueza de odonatos. Incrementar la abundancia y diversidad de macrófitas en los humedales muy posiblemente ayudaría a mantener la diversidad de odonatas del campus.

Palabras clave: Andes, Ecología urbana, Inventario, Sabana de Bogotá.

\begin{abstract}
The Sabana de Bogotá landscape has been highly modified and there are artificial wetlands that have been created for different purposes. Very little is known about the biodiversity of these wetlands. Objective: To compare the composition and species richness of Odonata in three artificial wetlands on the Universidad Militar Nueva Granada campus in Cajicá, Cundinamarca, Colombia. Methodology: Adults were captured using entomological nets during March and April, 2015. Three sites were sampled: 1. A wetland near the Bogotá river, 2. A temporary wetland surrounded by grassland, and 3. A wetland bordered by buildings and grassland and with the bottom covered with geomembrane. Results: 817 individuals belonging to the following species were collected: Rhionaeschna marchali (Aeshnidae), Enallagma civile (Coenagrionidae), Ischnura cruzi (Coenagrionidae), Ischnura sp. Mesamphiagrion laterale (Coenagrionidae) and Erythrodiplax fusca (Libellulidae). The riverine and temporary wetlands shared four species and showed greater similarity between them than with the geomembrane wetland. There was an association between the frequency of species of Odonata and each of the wetlands; for example; a species was unique to the riverine wetland (R. marchali) and another was exclusive to the temporary wetland (Ischnura sp.). There were no differences in estimated species richness between the riverine and temporary wetlands, and both
\end{abstract}

1 Programa de Biología Aplicada, Facultad de Ciencias Básicas y Aplicadas, Universidad Militar Nueva Granada, Cajicá, Colombia. e-mail: josecuellar1094@gmail.com

2 Programa de Biología, Facultad de Ciencias Básicas e Ingeniería, Universidad de los Llanos, Villavicencio, Colombia. Fecha recepción: Diciembre 16, $2015 \quad$ Fecha aprobación: Abril 17, $2016 \quad$ Editor Asociado: Torres-Torres M. 
had greater richness that the geomembrane wetland. Conclusion: Our results suggest that the differences between the wetlands affected the composition, frequency and richness of Odonata. Increasing the abundance and diversity of macrophytes should help to maintain the diversity of Odonata on campus.

Keywords: Andes, Inventory, Sabana de Bogotá, Urban ecology.

\section{Introducción}

En un sentido amplio, los humedales son zonas de la superficie terrestre que están temporal o permanentemente inundadas, y por lo tanto, su dinámica está regulada sobre todo por factores climáticos (van der Valk 2006). En los Andes colombianos y particularmente en la Sabana de Bogotá, los humedales naturales son ecosistemas amenazados como consecuencia de la expansión rural y urbana (Andrade 1998, van der Hammen et al. 2008), y poco es lo que se sabe sobre su composición biológica y ecología, lo que dificulta su manejo y conservación (Anónimo 2003, van der Hammen et al. 2008). Entre los elementos típicos de los humedales se destacan los odonatos (libélulas y caballitos del diablo) que son depredadores en todos sus estadios de desarrollo y además son parte de la dieta de otros animales, por lo que suelen ser componentes importantes en la red trófica de estos ecosistemas (Amat-García y Blanco-Vargas 2003). Los odonatos pueden controlar las poblaciones de otros organismos, incluyendo plagas de cultivos o vectores de enfermedades (González-Soriano 1993). Además, los odonatos se pueden usar como bioindicadores del estado de los humedales (Junior et al. 2015), porque los adultos pueden ser sensibles a las perturbaciones, lo que afecta su distribución y abundancia de especies (Kutcher y Bried 2014). En efecto, se ha encontrado que la presencia de odonatos es influida por distintas características del hábitat como la cobertura vegetal y en particular, las macrófitas (Urban 2004), que son utilizadas como refugio y sitios para la oviposición.

Los humedales de la Sabana de Bogotá se encuentran intervenidos por el ser humano (Mendivelso-López et al. 2007). Además, en la cuenca del río Bogotá se han construido humedales artificiales que funcionan como reservorios para actividades agropecuarias, o para manejar afluentes del río o con fines estéticos y deportivos. Si bien varios autores sugieren que los humedales artificiales pueden ayudar a la preservación de la biodiversidad (EPA 1993, Shutes 2001, Ehrenfeld 2004), hasta donde se ha estudiado, este aspecto no ha sido examinado en relación con los artrópodos de la Sabana de Bogotá. Con respecto al orden Odonata, en los humedales dentro de la ciudad de Bogotá y sus alrededores se han reportado las familias Aeshnidae, Libellulidae y Coenagrionidae (Amat-García y Quitiaquez 1998, Amat-García y Blanco-Vargas 2003, Sánchez-N y Amat-García 2005, Clavijo-Awazacko y Amarillo-Suárez 2013). También se ha propuesto que las características del humedal afectan la comunidad de odonatos (Kutcher y Bried, 2014). Con base en todo lo anterior, en este trabajo se explora de manera preliminar la comunidad de odonatos en tres humedales artificiales en Cajicá, Cundinamarca.

\section{Metodología}

Área de estudio. El campus de la Universidad Militar Nueva Granada (UMNG) ubicado en el municipio de Cajicá, Cundinamarca (Figura 1), 456'33.39" $\mathrm{N}-74^{\circ} 0{ }^{\prime} 46.24^{\prime \prime} \mathrm{O}, \sim 2550 \mathrm{~m}$ de elevación, cuenta con $\sim 76$ ha y está rodeado por zonas dedicadas a la agricultura, a la minería y campos de golf. El campus se ubica en un contexto suburbano y presenta pastizales dominados por Cenchrus clandestinus en aproximadamente en $70 \%$ de su área (Figura 2). Los ecosistemas naturales de la zona son humedales y bosques andinos (van der Hammen 2003). El campus colinda con el río Bogotá y es un ambiente altamente intervenido (Sánchez et al. 2015b). El campus solía usarse para la producción de ganado bovino y flores y por ello existen humedales artificiales asociados con el río Bogotá. Desde que se estableció la UMNG se han incrementado las edificaciones y se crearon, adicionalmente humedales artificiales que forman parte de un sistema para canalizar aguas. También se han hecho humedales temporales que se espera formen parte de un campo de golf.

Se hicieron muestreos en tres humedales. Humedal ronda de río que es producto de la construcción de jarillones que separaron el plano inundable del río Bogotá. Allí los sauces (Salix humdoldtiana) son abundantes y hay poca actividad humana comparado con los demás humedales del campus. La flora se encuentra caracterizada por la presencia de Bidens lae- 


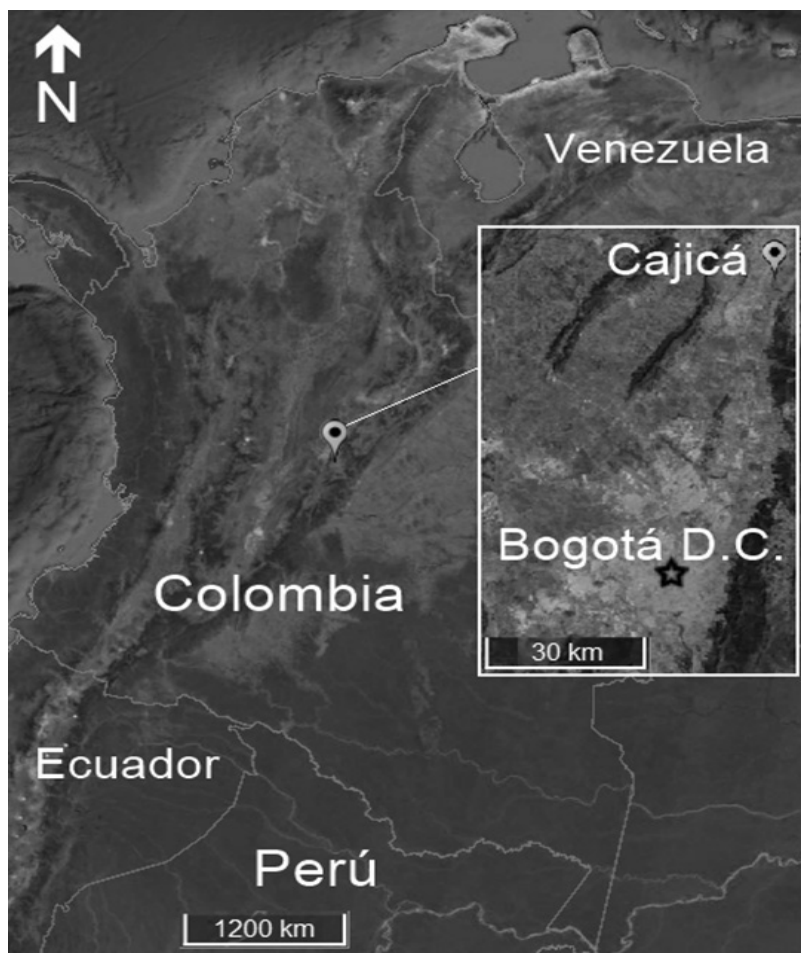

Figura 1. Ubicación del área de estudio en el municipio de Cajicá, departamento de Cundinamarca, Colombia.

vis, Hydrocotyle ranunculoides, Azolla filiculoides, Schoenoplectus californicus, Dryopteris wallichiana, Juncus effusus, Lemna gibba, Phytolacca bogotensis, Holcuslanatus, Polygonum hydropiperoides, Typha angustifolia, Verbena rondais, Eichhornia crassipes, C. clandestinus, Limnobium laevigatum. El segundo humedal es de tipo temporal (humedal temporal), resultado de excavaciones realizadas para la construcción de un campo de golf en 2014, en un área de pastizales dominada por C. clandestinus. Durante los muestreos presentó un espejo de agua temporal durante un mes, rodeado de S. californicus, J. effusus, H. lanatus, Potamogeton illinoensis de $\sim 1 \mathrm{~m}$ de altura. El tercer humedal presenta una geomembrana que recubre su fondo y está rodeado por edificios y pastizales dominados por C. clandestinus. Además, se observan Dypsis lutescens y Holcus lanatus, y plantas exóticas como Cyperus papyrus y Eriobotrya japonica.

Muestreo. La colecta de odonatos se efectuó durante marzo y abril del 2015. Durante marzo 2015 , la temperatura máxima del aire fue $19,5^{\circ} \mathrm{C}$, la temperatura mínima $9,5{ }^{\circ} \mathrm{C}$, y hubo una precipitación diaria entre 14 y $20 \mathrm{~mm}$, mientras que en abril, la temperatura máxima del aire fue $18^{\circ} \mathrm{C}$, la

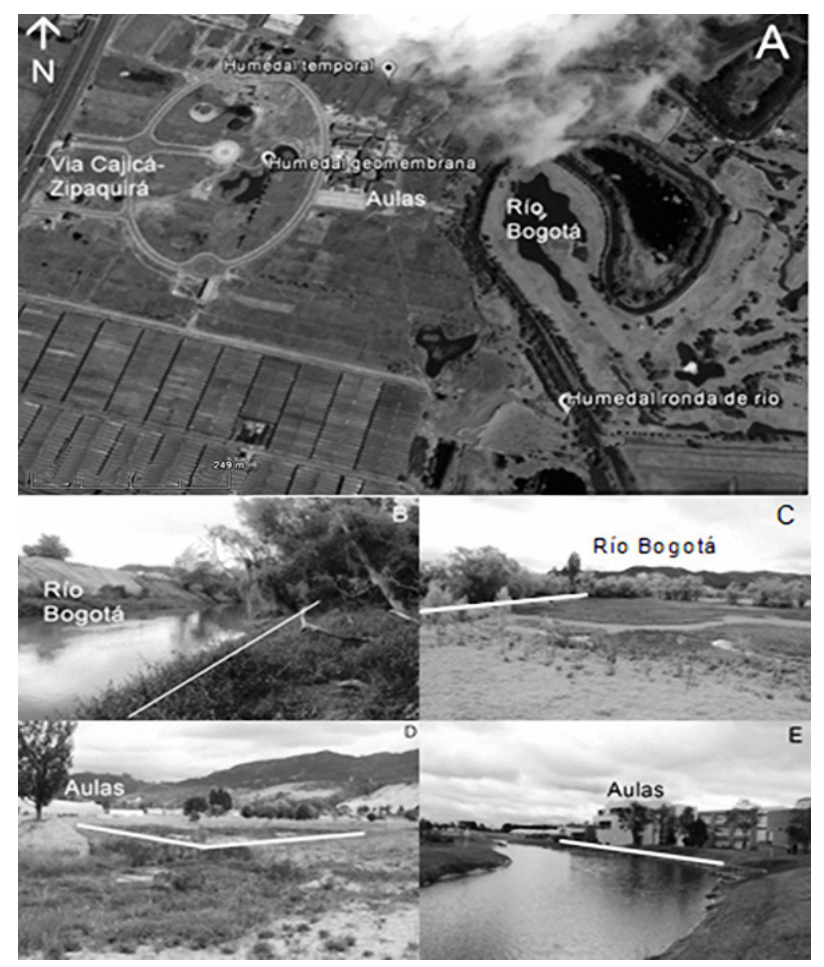

Figura 2. Humedales artificiales muestreados en el campus de la Universidad Militar Nueva Granada en Cajicá. A. humedal en la ronda del río Bogotá. B. C. humedal temporal. D. Humedal con geomembrana, E. Las líneas blancas indican los sitios de muestreo.

mínima $9,5^{\circ} \mathrm{C}$, y hubo una precipitacióndiaria entre 18 y $24 \mathrm{~mm}$ (datos de la estación meteorológica del campus). Se llevaron a cabo muestreos semanales entre las 12:30 h y 13:00 h, por ser el momento de mayor actividad para los adultos (Córdoba-Aguilar 2008). La colecta se realizó en recorridos de $100 \mathrm{~m}$, con redes entomológicas con un diámetro de $40 \mathrm{~cm}$ y profundidad de $70 \mathrm{~cm}$. En una primera sesión de tres horas se estandarizaron los métodos de colecta entre los investigadores. En las siguientes sesiones se hicieron dos muestreos semanales durante cinco semanas para un total de diez muestreos por humedal, con una duración de 30 min por cada uno, para un esfuerzo de muestreo total por humedal de cinco horas. De las recolecciones iniciales se obtuvieron especímenes de referencia que se llevaron al laboratorio. Los especímenes recolectados se sacrificaron con acetato de etilo y se preservaron los especímenes en montaje en seco con silica gel y su respectiva etiqueta de identificación, y se depositaron en la colección entomológica de la UMNG-Cajicá (números de catálogo 002634 al 002704). La identificación taxonómica se llevó a 
cabo hasta la mayor resolución posible con base en Garrison et al. (2006), Garrison y von Ellenrieder (2009), y Pérez y Palacino (2011).

Análisis de datos. Se realizó un análisis de agrupación basado en el índice de Jaccard, utilizando el algoritmo paired-group con PAST 3.0.1, con base en la presencia/ausencia de las especies en cada humedal. Para examinar la relación entre la frecuencia de cada especie en los tres humedales se realizó una prueba de $X^{2}$ con R 3.2.0 (Gómez-Anaya 2008). La riqueza de especies se calculó por el método de rarefacción y se estimó la riqueza total de cada humedal con el estimador no paramétrico de Chao 2 usando EstimateS 9.1.0 (Colwell 2013). Se escogió $\alpha=0,05$ como nivel de significancia para evaluar las hipótesis estadísticas.

\section{Resultados}

En los muestreos se capturaron seis especies pertenecientes a tres familias: Rhionaeschna marchali (Rambur 1842) (Aeshnidae), Erythrodiplax fusca (Rambur 1842) (Libellulidae), Enallagma civile (Hagen 1861) (Coenagrionidae), Ischnura cruzi (Coenagrionidae), Ischnura sp. (Coenagrionidae), y Mesamphiagrion laterale (Selys 1876) (Coenagrionidae). El humedal de ronda de río y el temporal compartieron cuatro especies y presentaron mayor similitud entre ellos que con el humedal con geomembrana (Figuras 3 y 4 ). La especie $R$. marchali fue recolectada únicamente en el humedal de ronda de río, Ischnura sp. se registró sólo en el humedal temporal, y el humedal con geomembrana no tuvo especies exclusivas. Las tres especies que se encontraron en el humedal con geomembrana estuvieron presentes también en los otros sitios muestreados.

Se encontró una asociación entre la frecuencia de especies de odonatos y los humedales estudiados $\left(X^{2}=281.1\right.$, g.l. $\left.=10, p<0.05\right)$. En el humedal con geomembrana, se presentaron en mayor frecuencia M. laterale y E. civile (Figura 4), y fueron además las más abundantes en las otras dos áreas. La especie más frecuente en todo el estudio fue E. civile (364 individuos), seguida de M. laterale (277 individuos). Las especies I. cruzi, Ischnura sp. y E. fusca se encontraron con mayor frecuencia en el humedal temporal. El género Ischnura, representado por dos especies (I. cruzi, 92 individuos y Ischnura sp., 61 individuos) se

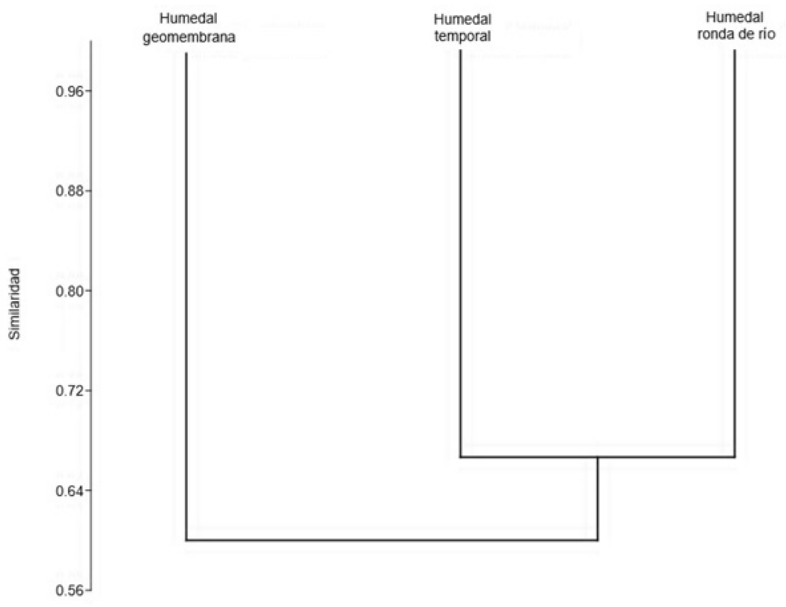

Figura 3. Análisis de similitud de tres humedales artificiales en el campus de la Universidad Militar Nueva Granada en Cajicá basados en su composición de especies.

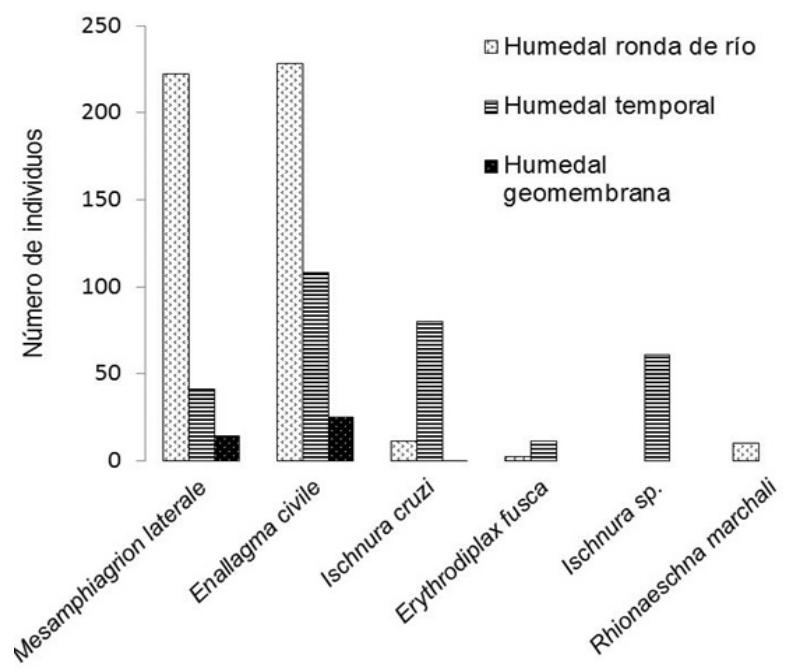

Figura 4. Frecuencia por especie de odonatos en tres humedales artificiales de la Universidad Militar Nueva Granada en Cajicá.

encontraron principalmente en el humedal temporal, y sólo algunas capturas ocurrieron en el humedal de ronda de río. La especie E. fusca presentó pocos individuos (13) localizados sobre todo en el humedal temporal. Por último, $R$. marchali (10 individuos), sólo se encontró en el humedal de ronda de río.

Las curvas de rarefacción indicaron que no hubo diferencias significativas en la riqueza de odonatos entre el humedal de la ronda del río Bogotá y los otros dos humedales, mientras que el humedal temporal tuvo una riqueza mayor que la del humedal con geomembrana (Figura 5). Por otra parte, la riqueza 


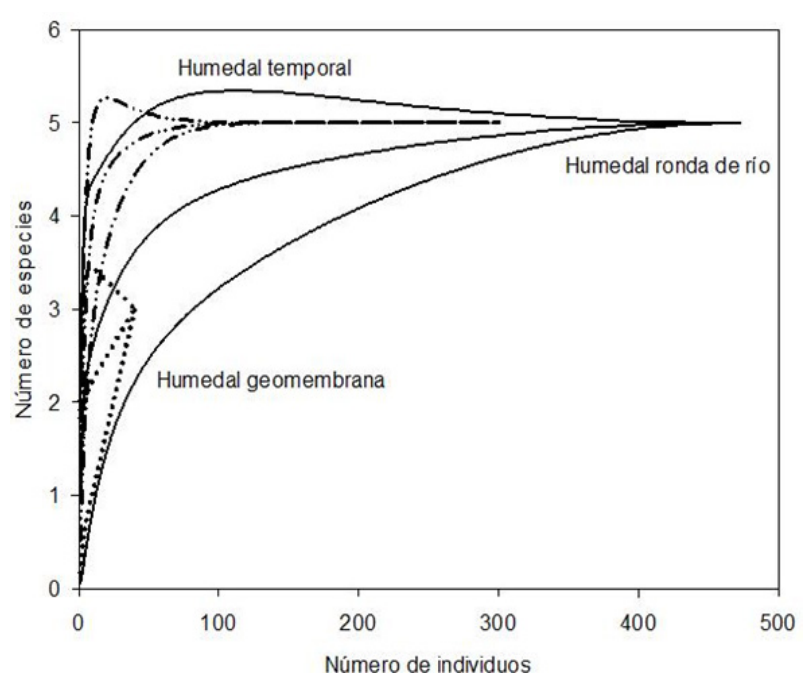

Figura 5. Curvas de acumulación de especies de tres humedales artificiales en la Universidad Militar Nueva Granada en Cajicá.

total estimada para el humedal con geomembrana [intervalo de confianza de Chao 2 (IC) 95\%: 1,71$3,20]$ fue significativamente menor que en los otros dos humedales y no hubo diferencias significativas entre el humedal del río (IC Chao 2 95\%: 3,9-5,10) y del humedal temporal (IC Chao 2 95\%: 4,57-5,00).

\section{Discusión}

La cantidad de especies (seis) y familias (tres) de Odonata en el área de estudio es similar a la reportada para otros sitios de la Sabana de Bogotá (Amat-García y Quitiaquez 1998, Amat-García y Blanco-Vargas 2003, Sánchez-N y Amat-García 2005, Clavijo-Awazacko y Amarillo-Suárez 2013). También, todas las especies encontradas en este trabajo han sido reportadas previamente en otros humedales de la Sabana de Bogotá y las únicas especies que no se registra en la UMNG fueron: Ischnura chingaza (Realpe 2009) y Sympetrum gilvum (Latorre-Cortés 2010).

El humedal con geomembrana fue el que menos macrófitas presentó en sus orillas y estaba en el contexto más perturbado por la presencia de construcciones. En éste se encontraron especies presentes en los humedales de ronda de río y temporal, pero en estos últimos hubo más especies y cada uno tuvo una especie exclusiva. Las especies presentes en el humedal con geomembrana fueron Mesamphiagrion laterale, Enallagma civile, Ischnura cruzi y han sido reportadas por Moreno-Pallares y Guillot-Monroy
(2012) como especies tolerantes a perturbaciones ambientales. La especie $M$. laterale, endémica de Venezuela y Colombia (Pulido 2014), fue dominante en el humedal con geomembrana, lo que ratifica su capacidad para explotar humedales perturbados. Por otra parte, la presencia de $R$. marchali únicamente en el humedal ronda de río sugiere que tal vez la menor intervención de este humedal favorece su presencia. Intrigante resulta que sólo se encontró a Ischnura sp. en el humedal temporal, que es un humedal de reciente creación, rodeado de pastizales y con poca vegetación propia de los humedales de la Sabana. Los especímenes de esta especie necesitan ser evaluados con más detalle para determinar su identidad taxonómica.

Por otra parte, R. marchali, Ischnura sp. y E. fusca fueron las especies más frecuentes en el humedal de ronda de río y el temporal, y esto posiblemente se deba a la presencia de diferentes macrófitas (Keiper et al. 2002, Alsfeld et al. 2009). El humedal de ronda de río y el temporal presentaron una mayor cantidad de individuos que el humedal con geomembrana. Particularmente, se ha sugerido que una mayor variedad de macrófitas se puede asociar con mayor disponibilidad y variedad de recursos alimentarios (von Ellenrieder 2003, Sánchez y Amat-García 2005).

La abundancia de odonatos se relaciona con la cantidad de presas, sitios de oviposición y percheo; esto a su vez depende de las características y abundancia de vegetación (González et al. 2009), lo que sugiere que los humedales artificiales menos intervenidos en el área de estudio tienen una mayor productividad lo que parece favorecer las poblaciones de varias especies de odonatos (Sánchez-N y Amat-García 2005, Clauznitzer et al. 2009). En efecto los resultados presentados aquí sugieren que la geomembrana al limitar el crecimiento de vegetación propia de humedales, reduce la riqueza y abundancia de odonatos. En este sentido, el manejo de este tipo de humedales artificiales se debería orientar hacia incrementar la presencia de macrófitas nativas (o incluso exóticas como el papiro) en las orillas e interior del humedal. Este tipo de modificaciones pueden potencialmente beneficiar no sólo a las libélulas, sino también para especies de aves nativas de los humedales de la Sabana (Sánchez et al. 2015a).

La relativamente alta riqueza de odonatos en el humedal temporal puede deberse a que es un ambiente 
que permite la llegada de odonatos en busca de lugares para alimentarse y reproducirse (Gibbons et al. 2002). Este lugar probablemente presenta sitios para el desarrollo, alimentación y reproducción asociados con las macrófitas allí presentes (Samways y Steytler 1996). Los odonatos son organismos que reaccionan a cambios del ambiente y colonizan nuevos lugares a través de la dispersión de individuos (Hart et al. 2014), además de ser controladores de poblaciones de diversos dípteros hematófagos. La abundancia y riqueza de odonatos en el humedal temporal sugiere que con poco manejo estos ambientes pueden incrementar la disponibilidad de hábitat para varias especies de odonatos y que por ello, el futuro campo de golf debería mantener las macrófitas propias de humedales en sus humedales asociados. También, el manejo adecuado de los humedales artificiales puede ayudar al control de las especies depredadas por los odonatos.

\section{Conclusiones}

Los resultados sugieren que algunas características de los humedales artificiales, como la abundancia y heterogeneidad vegetal, afectan la composición, frecuencia y riqueza de especies de odonatos en el campus de la UMNG. Se sugiere que estudios futuros examinen la variación de la composición, riqueza y abundancia de odonatos en relación con las características del agua y con mediciones de la vegetación, y del contexto que rodea a los humedales. Como el campus está en cambio y se planean hacer nuevas construcciones, se sugiere que en los humedales artificiales se mantenga o incremente la cobertura de macrófitas, lo que puede beneficiar a las libélulas y otras especies de fauna nativas de los humedales de la Sabana de Bogotá, y ayudar a que el campus esté en mayor armonía con el ambiente natural.

\section{Agradecimientos}

A la profesora María Isabel Castro por su constante apoyo a la investigación. A los laboratorios de la UMNG por el préstamo de los materiales para los muestreos y a la Vicerrectoría del Campus por permitir la realización del estudio. A Dinesh Rao por su revisión del Abstract.

\section{Literatura citada}

Alsfeld AJ, Bowman JL, Deller-Jacobs A. 2009. Effects of woody debris, microtopography, y organic matter amendments on the biotic community of constructed depressional wetlands. Biol Conserv. 142: 247-55.

Amat-García G, Blanco-Vargas E. 2003. Artropofauna de los humedales de la Sabana de Bogotá. En: Güarnizo A, Calvachi B (eds.). Los humedales de Bogotá y la Sabana. Bogotá: Acueducto de Bogotá, Conservación Internacional; pp. 90-106.

Amat-García G, Quitiaquez G. 1998. Un estudio de la entomofauna de humedales: el humedal Juan Amarillo en Bogotá. En: Guerrero E (ed.). Una aproximación a los humedales en Colombia. Bogotá: Fondo FEN Colombia, Unión Mundial para la Naturaleza; pp. 107-23.

Andrade GI. 1998. Los humedales del altiplano de Cundinamarca y Boyacá: ecosistemas en peligro de desaparecer. En: Guerrero E, Sánchez H, Alvarez R, Escobar EM (eds.). Una aproximación a los humedales en Colombia. Bogotá: Fondo FEN Colombia, Comité Colombiano de la UICN, UICN Oficina Regional para América del Sur; pp. 59-72.

Anónimo. 2003. Los humedales de Bogotá y la sabana. I y II. Bogotá: Acueducto de Bogotá y Conservación Internacional.

Clavijo-Awazacko H, Amarillo-Suárez A. 2013. Variación taxonómica y funcional en la artropofauna asociada a comunidades vegetales en humedales altoandinos (Colombia). Rev Colomb Entomol. 39: 155-63.

Clauznitzer V, Kalkman VJ, Ram M, Collen B, Baillie JEM, Bedjanic M, et al. 2009. Odonata enter the biodiversity crisis debate: the first global assessment of an insect group. Biol Conservat. 142: 1864-9.

Colwell RK. EstimateS: Statistical estimation of species richness and shared species from samples. Version 9.1.0 User's Guide and application. (En línea) 2013 (acceso 23 de junio). Disponible en: http:// purl.oclc.org/estimates

Córdoba-Aguilar A. 2008. Dragonflies and damselflies, model organisms for ecological and evolutionary research. New York: Oxford University Press; 303 pp.

Ehrenfeld JG. 2004. The expression of multiple functions in urban forested wetlands. Wetlands. 24: 719-33.

EPA. 1993. Constructed wetlands for wastewater treatment and wildlife habitat: 17 case studies. Washington DC: Environmental Protection Agency; 276 pp.

Garrison RW, von Ellenrieder N, Louton JA. 2006. Dragonfly genera of the New World. Baltimore: The Johns Hopkins University Press; 383 pp.

Garrison RW, von Ellenrieder N. 2009. Odonata. En: Domínguez E, Fernández HR (eds.) Macroinvertebrados bentónicos sudamericanos. Tucumán: Sistemática y biología, Fundación Miguel Lillo; 457 pp.

Gibbons LK, Reed JM, Chew FS. 2002. Habitat requirements and local persistence of three damselfly species (Odonata: Coenagrionidae). J Insect Conservat 6: 47-55.

Gómez-Anaya J. 2008. Ecología de los ensambles de larvas de odonatos (Insecta) y su uso potencial como indicadores de calidad ecológica en la Sierra. (Trabajo de Grado). 
Michoacán: Universidad Autónoma del estado de Hidalgo; $332 \mathrm{pp}$.

González-Soriano E. 1993. Odonata de México: situación actual y perspectivas de estudio. Revista de la Sociedad Mexicana de Historia Natural. 44: 291-303.

González-Soriano E, Noguera FA, Zaragoza-Caballero S, Ramírez-García E. 2009. Odonata de un bosque tropical caducifolio: sierra de San Javier, Sonora, México. Revista Mexicana de Biodiversidad. 80: 341-8.

Hart LA, Bowker MV, Tarboton W, Downs CT. 2014. Species composition, distribution and habitat types of odonata in the Simangaliso wetland park, KwaZulu-Natal, South Africa and the associated conservation implications. PLoS ONE. 9 (3): e92588.

Junior CDSM, Juen L, Hamada N. 2015. Analysis of urban impacts on aquatic habitats in the central Amazon basin: Adult odonates as bioindicators of environmental quality. Ecological Indicators. 48: 303-11.

Keiper JB, Walton WE, Foote BA. 2002. Biology and ecology of higher Diptera from freshwater wetlands. Ann Rev Entomol. 47: 207-32.

Kutcher TE, Bried JT. 2014. Adult Odonata conservatism as an indicator of freshwater wetland condition. Ecological Indicators. 38: 31-9.

Latorre-Cortés L. 2010. Relación de la entomofauna asociada a la descomposición de cuerpos de cerdo con el tiempo de sumersión posmortem (ISPM) en un ecosistema lentico de la sabana de Bogotá. (Tesis de Maestría). Bogotá: Facultad de Ciencias, Universidad Nacional de Colombia; 81 pp.

Mendivelso-López D, Navas-Camacho O, Padilla-Acosta J, Velásquez E. 2007. En: Hermelin M (ed.). Entorno natural de 17 ciudades de Colombia. Medellín: Fondo Editorial Universidad EAFIT; pp. 23-50.

Moreno-Pallares MI, Guillot-Monroy GH. 2012. Distribución espacial y temporal de náyades de odonatos en los humedales La Vaca y Santa María del Lago, Bogotá, Colombia. Acta Biol Colomb. 17: 281-94.

Pérez LA, Palacino F. 2011. Updated checklist of the odonata known from Colombia. Odonatologica. 40: 203-25.

Pulido L. 2014. Etapas de madurez sexual identificadas por coloración en Mesamphiagrion laterale (Zygoptera: Coenagrionidae). (Trabajo de Grado). Bogotá: Facultad de Ciencias, Universidad El Bosque; 102 pp.

Realpe E. 2009. Diversidad del género Ischnura (Odonata: Coenagrionidae) y su relación con la altitud y orogenia de la Cordillera Oriental-Andes colombianos. (Tesis Doctoral). Bogotá: Facultad de Ciencias, Universidad de los Andes; 102 pp.

Samways MJ, Steytler NS. 1996. Dragonfly (Odonata) distribution patterns in urban and forest landscapes, and recommendations for riparian management. Biological Conservation. 78: 279-88.

Sánchez F, Casallas M, Bobadilla G. 2015a. Abundancia y reproducción de Porphyriops melanops en un humedal artificial suburbano en Bogotá, Colombia. Ornitol Colomb 15: e10-e18.

Sánchez F, Martínez-Habibe MC, Díaz S, Medina N, Riaño J, PaQui MF. 2015b. Biodiversidad en un campus universitario en la Sabana de Bogotá: inventario de plantas y tetrápodos. Boletín Cientifico del Centro de Museos - Universidad de Caldas 19: 186-203.

Sánchez-ND, Amat-García GD. 2005. Diversidad de la fauna de artrópodos terrestres en el humedal Jaboque, Bogotá, Colombia. Caldasia. 27: 311-29.

Shutes RBE. 2001. Artificial wetlands and water quality improvement. Environ Internat. 26: 441-7.

Urban MC. 2004. Disturbance heterogeneity determines freshwater metacommunity structure. Ecology 85: 2971-8.

van der Hammen T. 2003. Los humedales de la Sabana: origen, evolución, degradación y restauración. En: Güarnizo A, Calvachi B (eds.). Los humedales de Bogotá y la Sabana. Bogotá: Acueducto de Bogotá, Conservación Internacional; pp. 19-51.

van der Hammen T, Stiles FG, Rosselli L, Chisacá-Hurtado LM, Ponce de Léon GC, Guillot-Monroy G, et al. 2008. Protocolo de recuperación y rehabilitación ecológica de humedales en centros urbanos. Bogotá: Secretaría Distrital de Ambiente, Alcaldía Mayor de Bogotá; 356 pp.

van der Valk AG. 2006. The biology of greshwater wetlands biology of habitats. New York: Oxford University Press Inc.

von Ellenrieder N. 2003. A synopsis of the neotropical species of 'Aeshna' Fabricius: The genus Rhionaeschna Förster (Odonata: Aeshnidae). Tijdschriftvoor Entomologie. 146: 67-207. 